\title{
Novel cytotoxic brominated diterpenes from the red alga Laurencia obtusa
}

Dimitra Iliopoulou, ${ }^{\dagger}$ Nikos Mihopoulos, ${ }^{\ddagger}$ Constantinos Vagias, ${ }^{\dagger}$ Panagiota Papazafiri,
and Vassilios Roussis $*, \dagger$

\section{Supplementary Information Index}

Page

S2 Figure $\mathrm{S} 1{ }^{1} \mathrm{H} \&{ }^{13} \mathrm{C}$ spectrum of prevezol C (1)

S3 Figure $\mathrm{S} 2{ }^{1} \mathrm{H} \&{ }^{13} \mathrm{C}$ spectrum of prevezol B (2)

S4 Figure $\mathrm{S} 3{ }^{1} \mathrm{H} \&{ }^{13} \mathrm{C}$ spectrum of prevezol D (3)

S5 Figure $\mathrm{S} 4{ }^{1} \mathrm{H} \&{ }^{13} \mathrm{C}$ spectrum of prevezol E (4)

S6 Figure $\mathrm{S} 5{ }^{1} \mathrm{H} \&{ }^{13} \mathrm{C}$ spectrum of neorogioldiol (5)

S7 Figure $\mathrm{S} 6{ }^{1} \mathrm{H} \&{ }^{13} \mathrm{C}$ spectrum of neorogioldiol $\mathrm{B}(\mathbf{6})$

S8 Figure $\mathrm{S} 7{ }^{1} \mathrm{H} \&{ }^{13} \mathrm{C}$ spectrum of $O^{11}, 15$-cyclo-14-bromo-14,15-dihydrorogiol3,11-diol (7) 


\section{Compound 1 (Prevezol C)}
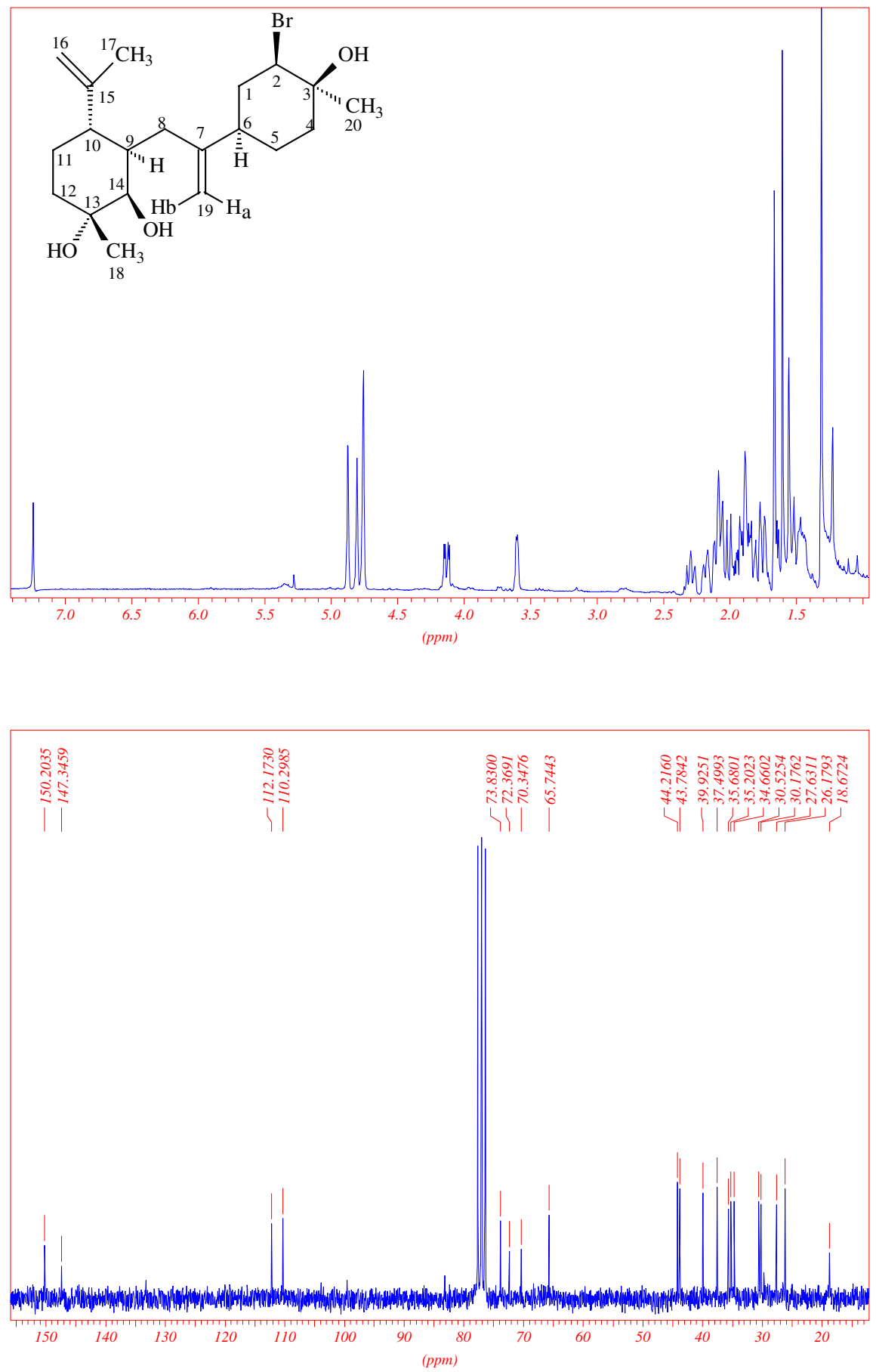

Figure S1. ${ }^{1} \mathrm{H} \&{ }^{13} \mathrm{C}$ spectrum of prevezol C (1) 


\section{Compound 2 (Prevezol B)}
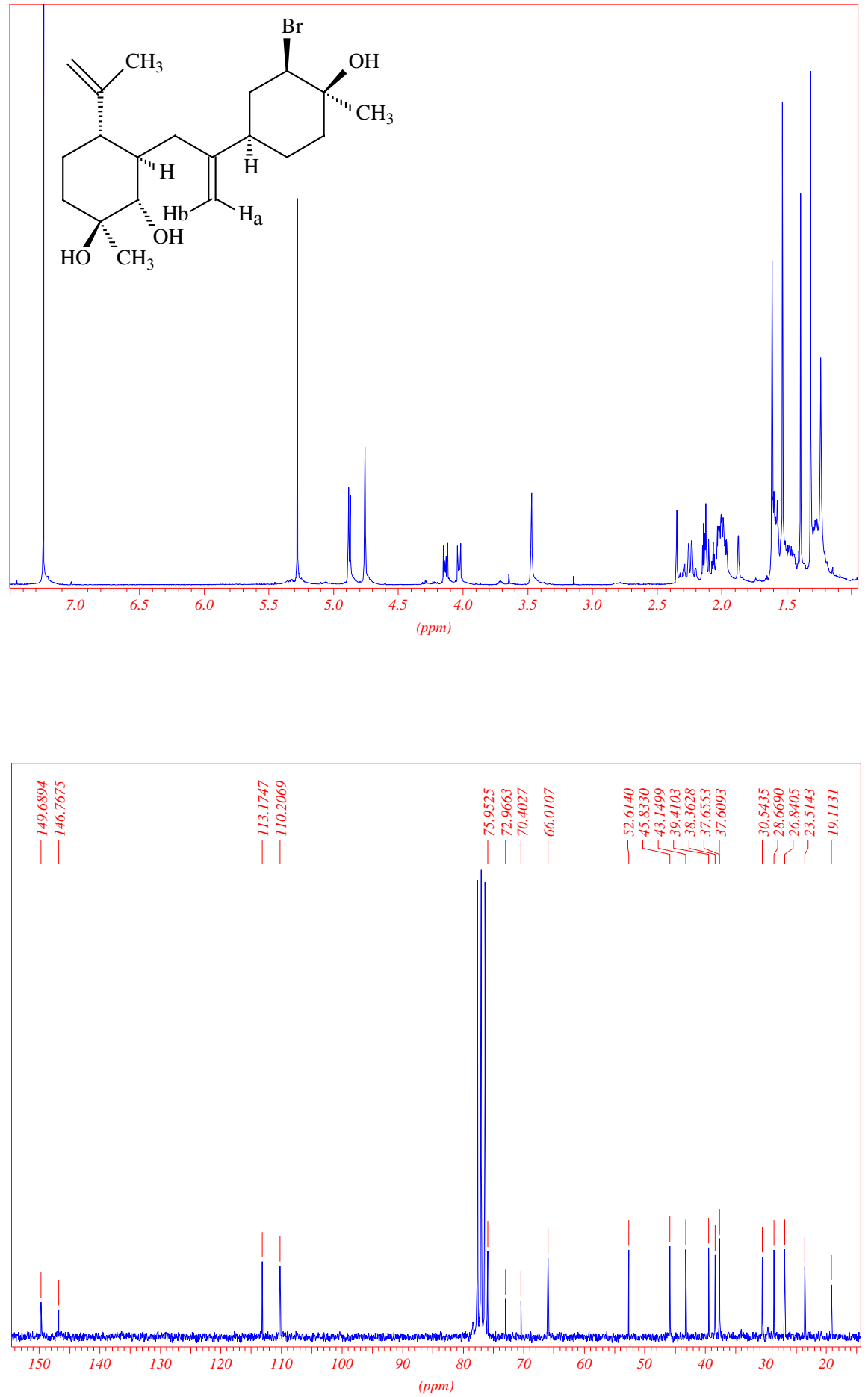

Figure S2. ${ }^{1} \mathrm{H} \&{ }^{13} \mathrm{C}$ spectrum of prevezol B (2) 


\section{Compound 3 (Prevezol D)}
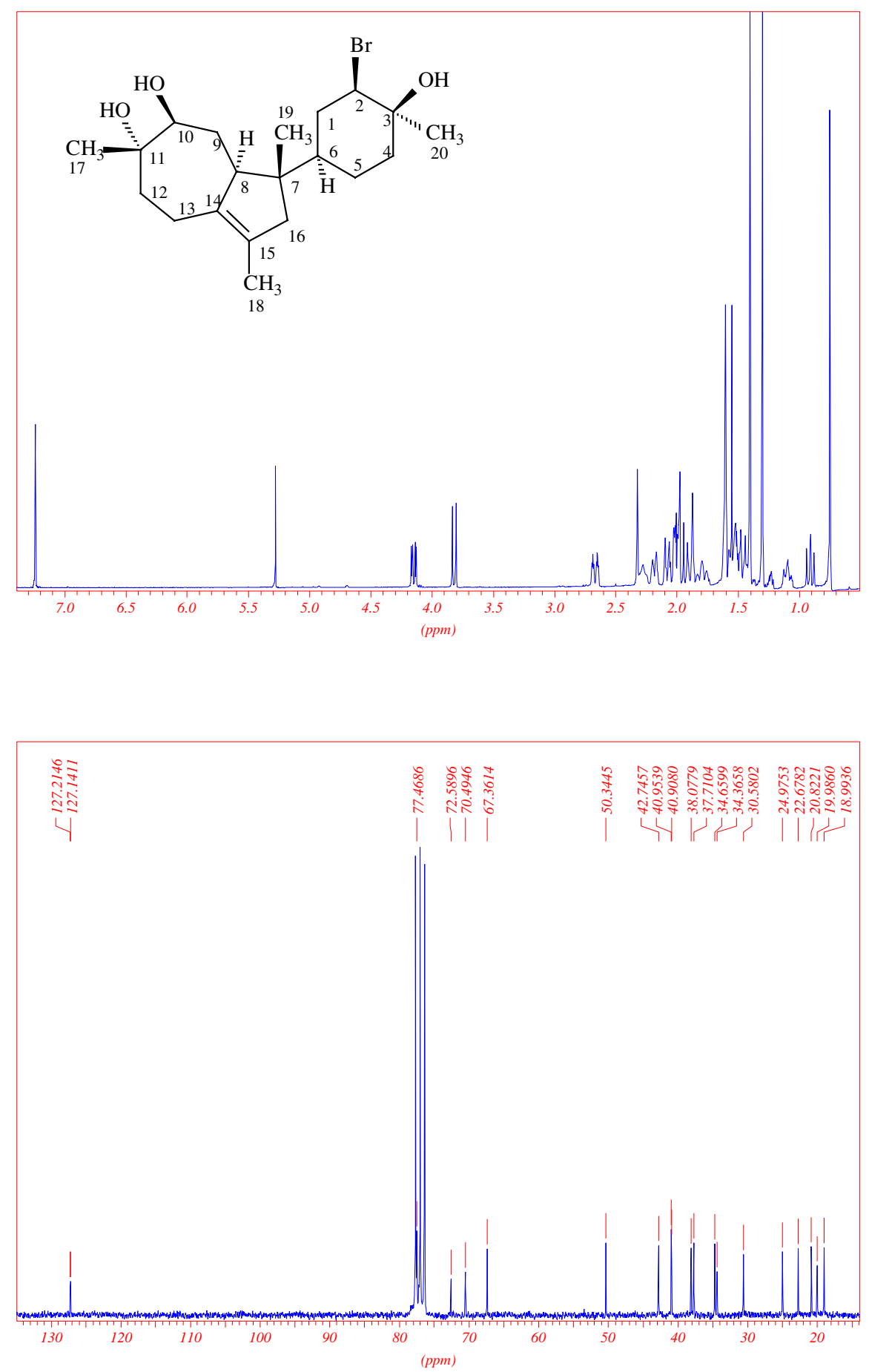

Figure S3. ${ }^{1} \mathrm{H} \&{ }^{13} \mathrm{C}$ spectrum of prevezol D (3) 


\section{Compound 4 (Prevezol E)}
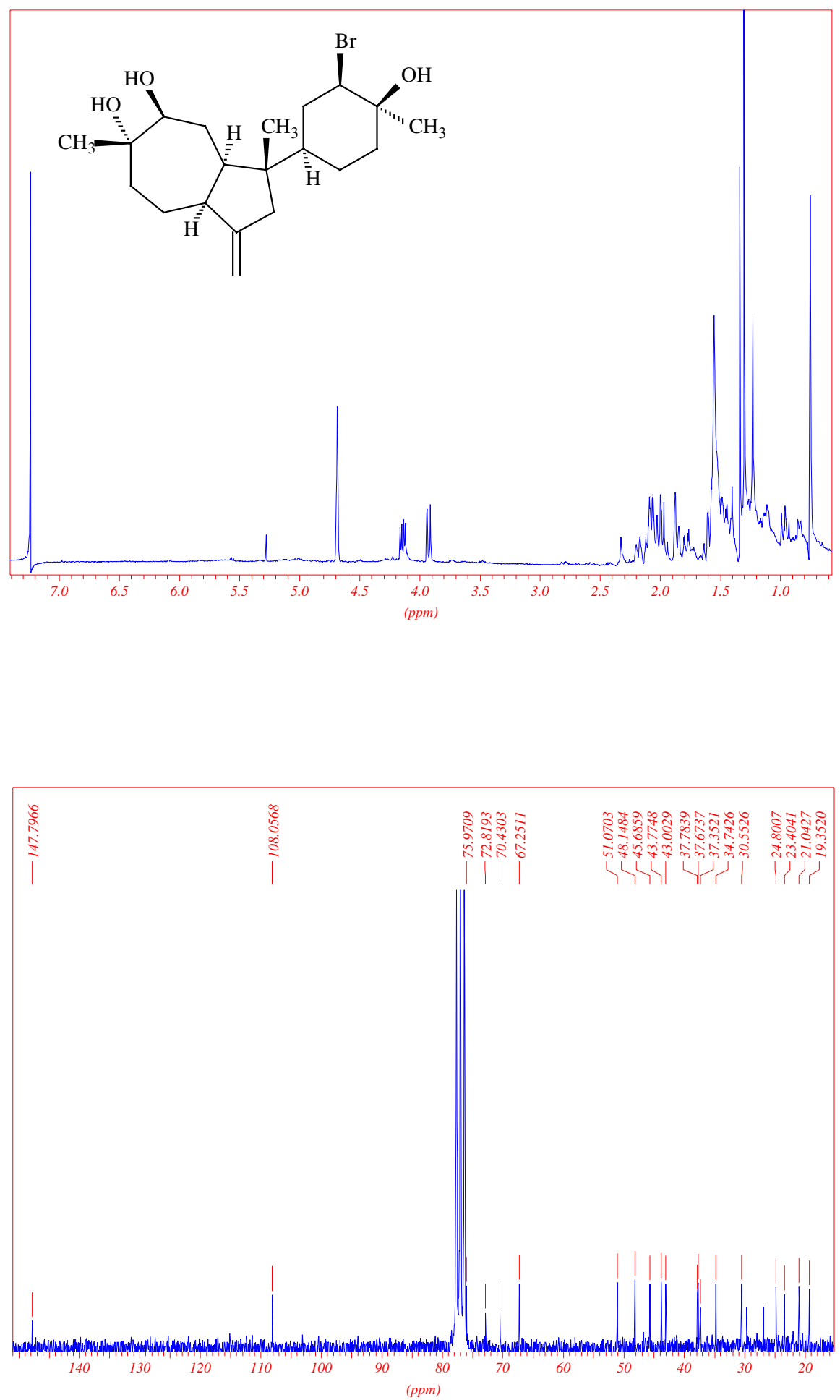

Figure S4. ${ }^{1} \mathrm{H} \&{ }^{13} \mathrm{C}$ spectrum of prevezol E (4) 


\section{Compound 5 (Neorogioldiol)}
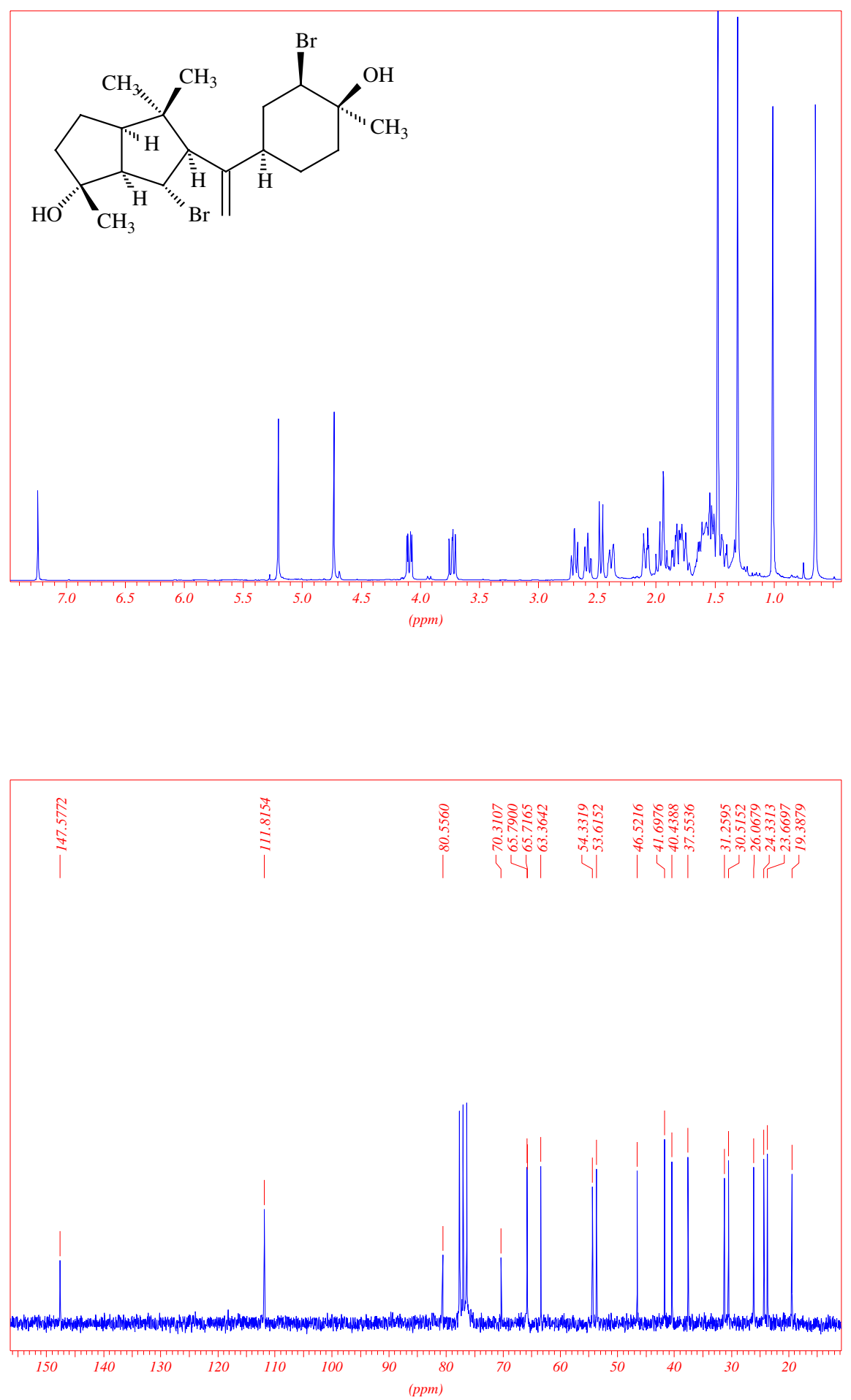

Figure S5. ${ }^{1} \mathrm{H} \&{ }^{13} \mathrm{C}$ spectrum of neorogioldiol (5) 


\section{Compound 6 (Neorogioldiol B)}
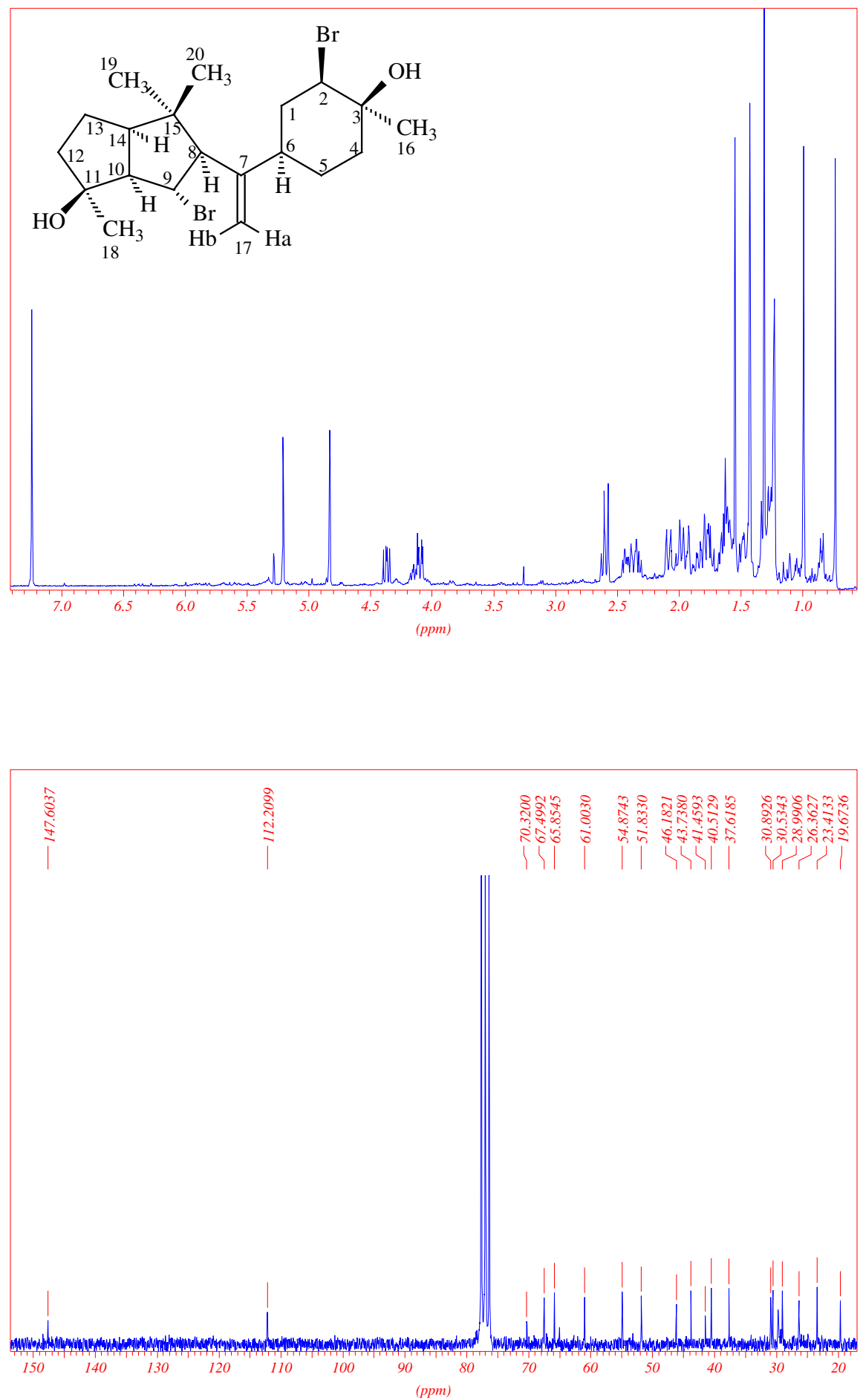

Figure S6. ${ }^{1} \mathrm{H} \&{ }^{13} \mathrm{C}$ spectrum of neorogioldiol B (6) 


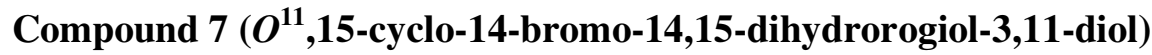
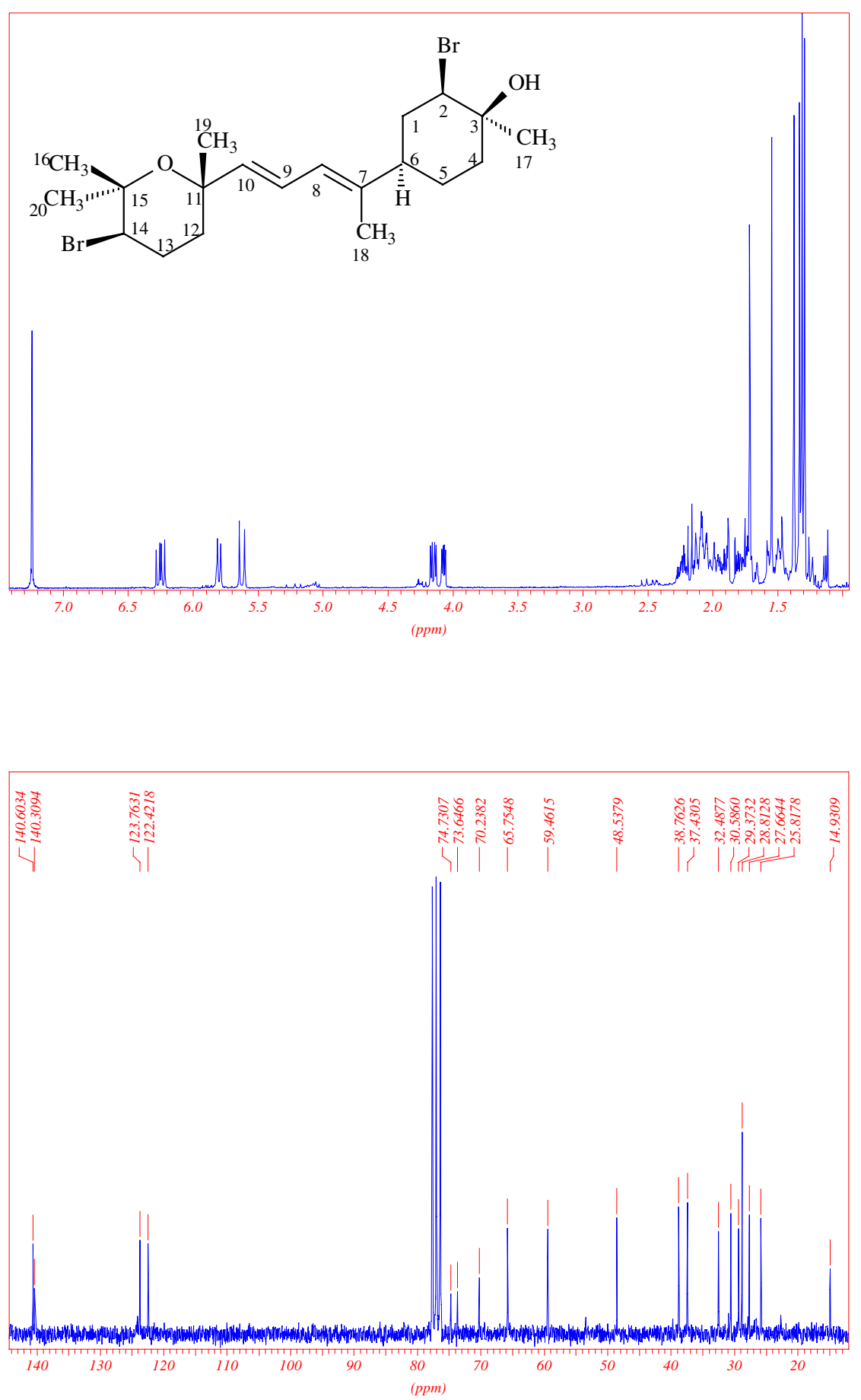

Figure S7. ${ }^{1} \mathrm{H} \&{ }^{13} \mathrm{C}$ spectrum of $O^{11}$,15-cyclo-14-bromo-14,15-dihydrorogiol-3,11diol (7) 